\title{
Inter-gender differences of balance indicators in persons $60-90$ years of age
}

This article was published in the following Dove Press journal:

Clinical Interventions in Aging

\section{Ewa Puszczalowska-Lizis' \\ Przemyslaw Bujas ${ }^{2}$ \\ Slawomir Jandzis' \\ Jaroslaw Omorczyk ${ }^{2}$ \\ Marek $\mathrm{Zak}^{3}$}

'Faculty of Medicine, Institute of Physiotherapy, University of Rzeszow, Rzeszow, Poland; ${ }^{2}$ Faculty of Physical Education and Sports, Institute of Sport, University School of Physical Education, Krakow, Poland; ${ }^{3}$ Faculty of Medicine and Health Sciences, Institute of Physiotherapy, The Jan Kochanowski University, Kielce, Poland
Correspondence: Marek Zak Faculty of Medicine and Health Sciences, Institute of Physiotherapy, The Jan Kochanowski University, ul. IX Wiekow Kielc 19, 25-317 Kielce, Poland

Tel +48 4I 3496909

Email mzak।@onet.eu
Introduction: Precision of movements responsible for maintaining balance deteriorates with age due to natural involutionary processes, thus prompting a research question whether the values of gender-related stability indicators might differ significantly among the study subjects over 60 years of age.

Methods: The study group comprised 136 seniors ( 89 women, 47 men; aged 60-90 years). The CQ-Stab 2P 2-platform posturograph was used as the main research device, whereas the Mann-Whitney $U$-test was used to evaluate the gender-related differences in the average level of variables.

Results: In the open-eye test, significant gender-related differences were observed with regard to the statokinesiogram's path length in the mediolateral (ML) direction in the subjects aged 60-69 years $(p=0.004)$, mean frequency of center of pressure (COP) displacement and number of COP displacements in the ML direction in the subjects aged $70-79$ years $(p=0.028$, $p=0.019$ ), and mean COP displacement in the anteroposterior (AP) direction in the subjects aged $80-90$ years $(p=0.026$ ). When the subjects were deprived of visual control, gender-related differences were observed with regard to the mean frequency of COP displacement, number of COP displacements in the ML direction in the subjects aged 60-69 years ( $p=0.045, p=0.049$ ), and the statokinesiogram's path length in the AP direction in the subjects aged 70-79 years ( $p=0.015$ ). In the oldest age group, the differences were noted in the statokinesiogram's path length in the AP direction ( $p=0.001)$, a sway area delimited by the COP point ( $p=0.003)$, range of AP stability $(p<0.001)$, and range of ML stability $(p=0.048)$.

Conclusion: Gender-related differences affecting postural stability were found in the elderly. Men were characterized by a lower level of postural stability when compared with women. This highlights the need to have the gender-related differences taken into account, when developing various preventive and therapeutic programs specifically aimed at compensating certain involution-dependent deficits.

Keywords: aged, balance, postural control, geriatrics

\section{Introduction}

Aging is an irreversible process that progresses at a slower or faster pace during ontogenesis. It is associated with the occurrence of involutionary changes that result in an impaired function of various bodily systems and/or organs. Involutionary processes affect the brain, sensory and internal organs, and the motor system. Issues pertaining to aging, and the underlying mechanisms have long been of interest to numerous investigators. Chen et $\mathrm{al}^{1}$ demonstrated that extended atherosclerotic changes in the cerebral cortex in elderly persons adversely affected intellectual ability and the speed of thinking. Biskup et $\mathrm{al}^{2}$ highlight that normal functioning of visual, auditory, tactile, motor, and other analyzers is subject to deterioration and impairment with age. 
The response to different situations may be delayed, inadequate, or altogether absent. Doherty ${ }^{3}$ argues that an elderly person gets tired more quickly, as reactions to various stimuli and perception at large require more effort. Decreased muscle strength and overall muscle atrophy result in an inability to produce adequate tension, which is necessary for all reflex reactions. Al-Momani et $\mathrm{al}^{4}$ revealed a significant impact of upper limb disability, stroke, heart disease, arthritis, joint diseases, diabetes, and hypertension, as well as psychiatric disorders and cognitive disabilities on balance deficits among elderly adults. Vaughan et $\mathrm{al}^{5}$ and Zak et $\mathrm{al}^{6}$ point out that old age is associated with frequent occurrence of pathological changes associated with pain and the need for immobilization, in conjunction with a therapy entailing analgesics, antidepressants, and tranquilizers. Owing to poor general condition stemming from periods of inactivity, poor physical form, and functional impairment of postural stability are inevitable. In the osteoarticular system, elasticity of articular cartilage, and of the connective tissue surrounding the joints, is appreciably reduced. Hsu et $\mathrm{al}^{7}$ highlight that decreased bone mass caused by osteoporosis results in the deformation of the vertebral body, promotes changes in the shape of spine, as well as may, to some extent, be responsible for bad posture and reduced lower limb mobility.

These factors are deemed conducive to the loss of balance and postural instability in the elderly. Cameron et $\mathrm{al}^{8}$ emphasized that instability, in the absence of adequate compensation, increased the likelihood of sustaining accidental falls. Melton ${ }^{9}$ and Francis ${ }^{10}$ established that women were more susceptible to falls than men, in line with the findings yielded by the epidemiological studies conducted on the nature of falls sustained by the elderly, that is, women were in fact 3 times more likely to fall, and twice as likely to suffer a fracture. Naessen et $\mathrm{al}^{11}$ and Otero et $\mathrm{al}^{12}$ consider the decrease in the levels of sex hormones, the main cause of postmenopausal osteoporosis, to be one of the causative factors in balance problems experienced by women in the fifth and sixth decades of their life.

According to Liu-Ambrose et $\mathrm{al}^{13}$ the underlying causes of instability in persons with osteoporosis are the fractures and distortions of the decalcified vertebrae, which account for the reshaping of the spine curvature and deformed body geometrics, consequently impacting overall balance of the forces that affect individual segments of the body. Furthermore, they also affect the progression of degenerative changes and account for their painfulness, which aggravate postural balance problems still further. In line with the findings of Naessen et al, ${ }^{11}$ menopausal symptoms caused by vasomotor disorders also adversely affect the ability to maintain balance. Ishikawa et $\mathrm{al}^{14}$ attribute instability to the changes in posture caused by deformed vertebrae and muscle weakness. Campbell et al, ${ }^{15}$ Frontera et $\mathrm{al}^{16}$ and Cooper et al ${ }^{17}$ emphasize that in older women decreased muscle strength, reaches its critical level earlier than in men; this subsequently translating into relatively faster degradation of proprioception, and, consequently, deterioration of postural balance. Menant et al ${ }^{18}$ and Cronin et al, ${ }^{19}$ on the other hand, attribute proprioceptive dysfunction in women to wearing less comfortable shoes, which are often too narrow, stiff, and high-heeled, in a group of subjects aged between 75 and 84 years.

Kado et $\mathrm{al}^{20}$ and Takahashi et $\mathrm{al}^{21}$ emphasize the differences in male and female posture. The authors claim the exacerbation of kyphosis with age to be a more common problem for women. According to some authors, changes in posture have a significant impact on the postural stability in the elderly. Sinaki et al $^{22}$ argue there is a greater likelihood of imbalance in the persons with exacerbated chest kyphosis that - when concurrent with an excessive tilt of the torso forward - causes the body's center of gravity (COG) to shift close to the front edge of the support plane.

Although steadily deteriorating precision of movements that ensure balance control is the result of natural involutionary processes that occur in both sexes, the question whether stability indicators differ significantly between women and men is yet to be conclusively answered. The present study aimed, therefore, to identify the gender-related differences in the selected indicators of balance observed in the subjects over the age of 60 years.

Findings yielded by our own research may well serve as a reference point for the evaluation and interpretation of the results of research into postural stability, as well as a good pointer with regard to mapping out preventive and therapeutic programs, including balance training in the elderly, primarily with a view to improving safety and overall quality of life in the elderly.

\section{Methods}

The study covered 136 free-living community dwellers aged 60-90 years. The subjects were randomly selected from among those who completed 60 years of age, were at a purpose-built, housing estate for the seniors, as well as were found fully compliant with the inclusion criteria for the study protocol. The following inclusion criteria were applied: age range of 60-90 years, dominating right hand and leg (determined on the basis of the Waterloo Handedness and Footedness Questionnaire - Revised ${ }^{23}$ ), level of physical fitness 
that facilitates walking without any orthopedic aids (canes, crutches, and walkers), ability to assume a standing position on the stabilographic platform without any assistance, and a written informed consent to participate in the study. Victims of any cerebral accidents, persons with hemiplegia, parkinsonism and those on medications that might affect balance, were duly excluded from the study.

Three age groups were considered. The first group consisted of the subjects aged 60-69 years, and comprised 17 women (mean age: $\overline{\mathrm{x}}=64.11 \pm 3.55$ years) and 13 men (mean age: $\bar{x}=65.86 \pm 3.08$ years). The second group (70-79 years) comprised 29 women (mean age: $\bar{x}=76.15 \pm 2.94$ years) and 14 men (mean age: $\bar{x}=74.19 \pm 2.69$ years). The oldest age group (80-90 years) consisted of 43 women (mean age: $\overline{\mathrm{x}}=85.96 \pm 4.38$ years) and 20 men (mean age: $\overline{\mathrm{x}}=85.54 \pm 3.80$ years $)$.

Stabilographic measurements were taken using the CQStab 2P 2-platform posturograph (CQ Electronic System). The device allowed simultaneous recording of the vertical center of pressure position of the forces affecting each foot. Data from 6 sensors (3 in each platform) were recorded. Sampling totaled $200 \mathrm{~Hz}$ per sensor. The platforms were leveled, their surfaces aligned within a single plane. All study subjects were furnished with detailed information on the aims and methods to be used throughout the study protocol. The study protocol featured two 30-second successive tests. After getting onto the platform, a person assumed a natural standing position (a quiet stance), with the upper limbs hanging along the body. The stance width and foot angle were natural, each foot resting on a separate panel of the platform.

The first test consisted of measuring the body's stability with the eyes wide open. The subject stood still, trying to maintain visual focus on the point of reference placed in front of him, at a distance of 1 meter, at eye level. The proper measurement was preceded by a 30 -second "training" stint to stabilize the balance, and then the test readings were recorded. Subsequently, the second test was conducted, while the subject had his eyes closed (ie, had no visual control over the positioning of his body).

During the tests, the investigator was always positioned behind the subject.

The motion signal, as generated by the point of application of the resultant pressure force exerted by the feet directly onto the platform plates, duly registered in the computer memory, was then used to calculate the values of the stability indicators. The names of the displacement directions of the center of foot pressure (COP) were referred to the Cartesian coordinate system, as made up by the pairs of perpendicular axes. The $\mathrm{X}$-axis was the axis of the abscissae, and the $\mathrm{Y}$-axis - of the ordinate, whereas the point $\mathrm{O}$ (coordinates 0.0 ) that is, the origin of the system of coordinates was the so-called "geometric COG of the COP trajectory".

The following indicators of stability were assessed:

- SPAP - statokinesiogram path length on the Y-axis (ie, the trajectory followed by the COP in the anteroposterior (AP) direction during a 30-second measurement), in $\mathrm{mm}$;

- SPML - statokinesiogram path length on the X-axis (ie, the trajectory followed by the COP in the mediolateral [ML] direction during a 30-second measurement), in $\mathrm{mm}$;

- SA - sway area delimited by the COP point (ie, the surface area of an irregularly shaped polygon, demarcated by a boundary line connecting up the extreme points of the statokinesiogram), in $\mathrm{mm}^{2}$;

- $\mathrm{MAAP}$ - mean COP displacement from the origin on the $\mathrm{Y}$-axis (in the AP direction) during a 30 -second measurement, in mm;

- MAML - mean COP displacement from the origin on the $\mathrm{X}$-axis (in the ML direction) during a 30-second measurement, in $\mathrm{mm}$;

- MaxAP - range of AP stability: maximal displacement of the COP from the origin on the $\mathrm{Y}$-axis (ie, maximal amplitude of the COP in the AP direction), in mm;

- MaxML - range of ML stability: maximal displacement of the COP from the origin on the $\mathrm{X}$-axis (ie, maximal amplitude of the COP in the ML direction), in $\mathrm{mm}$;

- $\mathrm{MF}$ - mean frequency of COP displacement (ie, the ratio of the total statokinesiogram's path length [on both axes] to the circumference of the circle the radius of which is equal to the average COP displacement, calculated per 1 second), in $\mathrm{Hz}$;

- LWAP - number of COP displacements along the Y-axis (ie, number of COP displacements in the AP direction, within the range: over $0.2 \mathrm{~mm}$ - below $-0.2 \mathrm{~mm}$, relative to the center of the coordinate system);

- LWML - number of COP displacements along the X-axis (ie, number of COP displacements in the ML direction, within the range: over $0.2 \mathrm{~mm}$ - below $-0.2 \mathrm{~mm}$, relative to the center of the coordinate system);

- The selection of stability indicators was based on the frequency of their use in the subject literature. ${ }^{24-27}$ Examples of the path length for the COP are comprised in Figures 1 and 2.

Anthropometric measurements of the body mass and height were taken to calculate the body mass index. The basic 


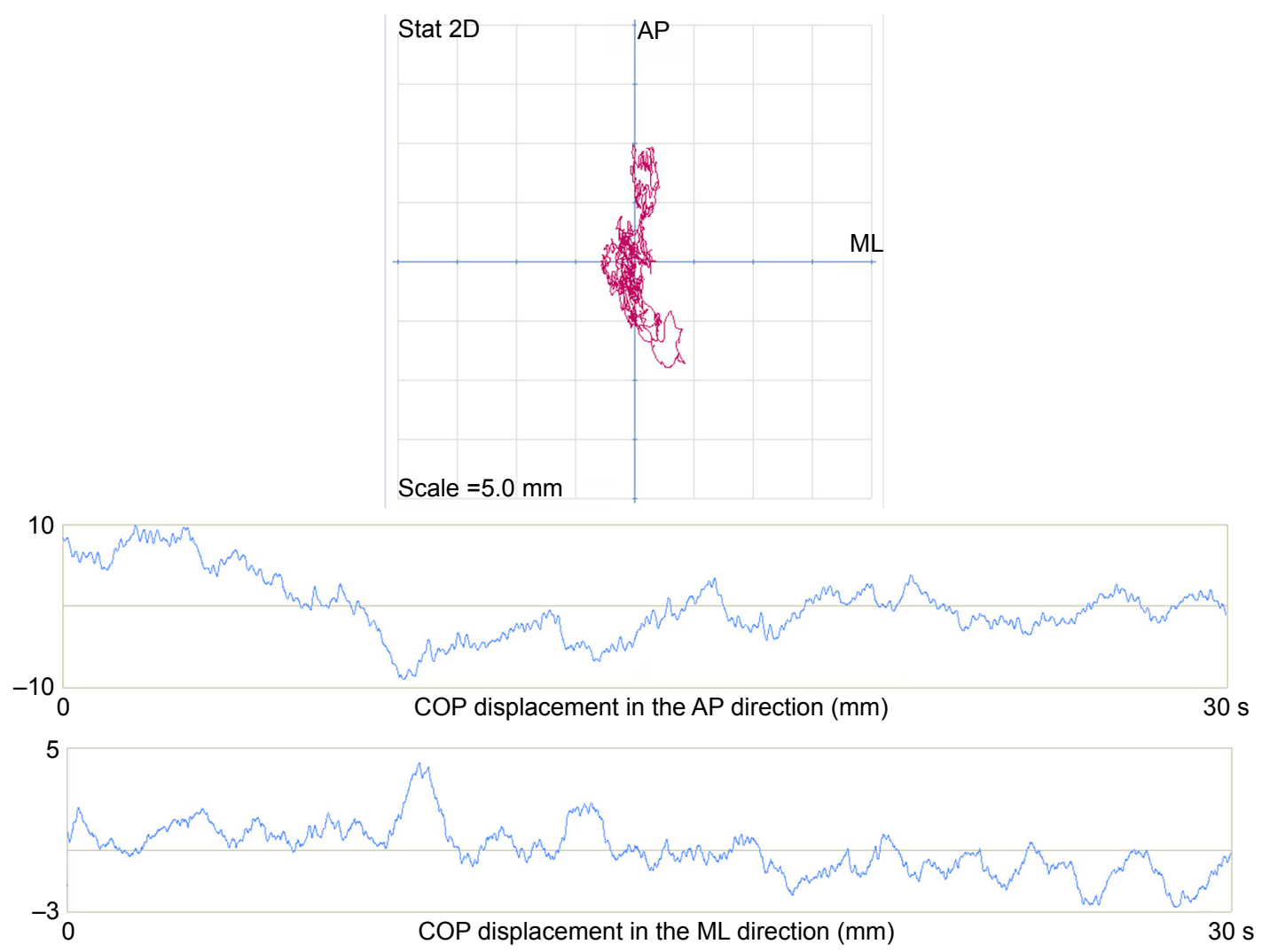

Figure I Diagram showing the sample of the path length for the COP during the test with the eyes open. Abbreviations: AP, anteroposterior; COP, center of pressure; ML, mediolateral.

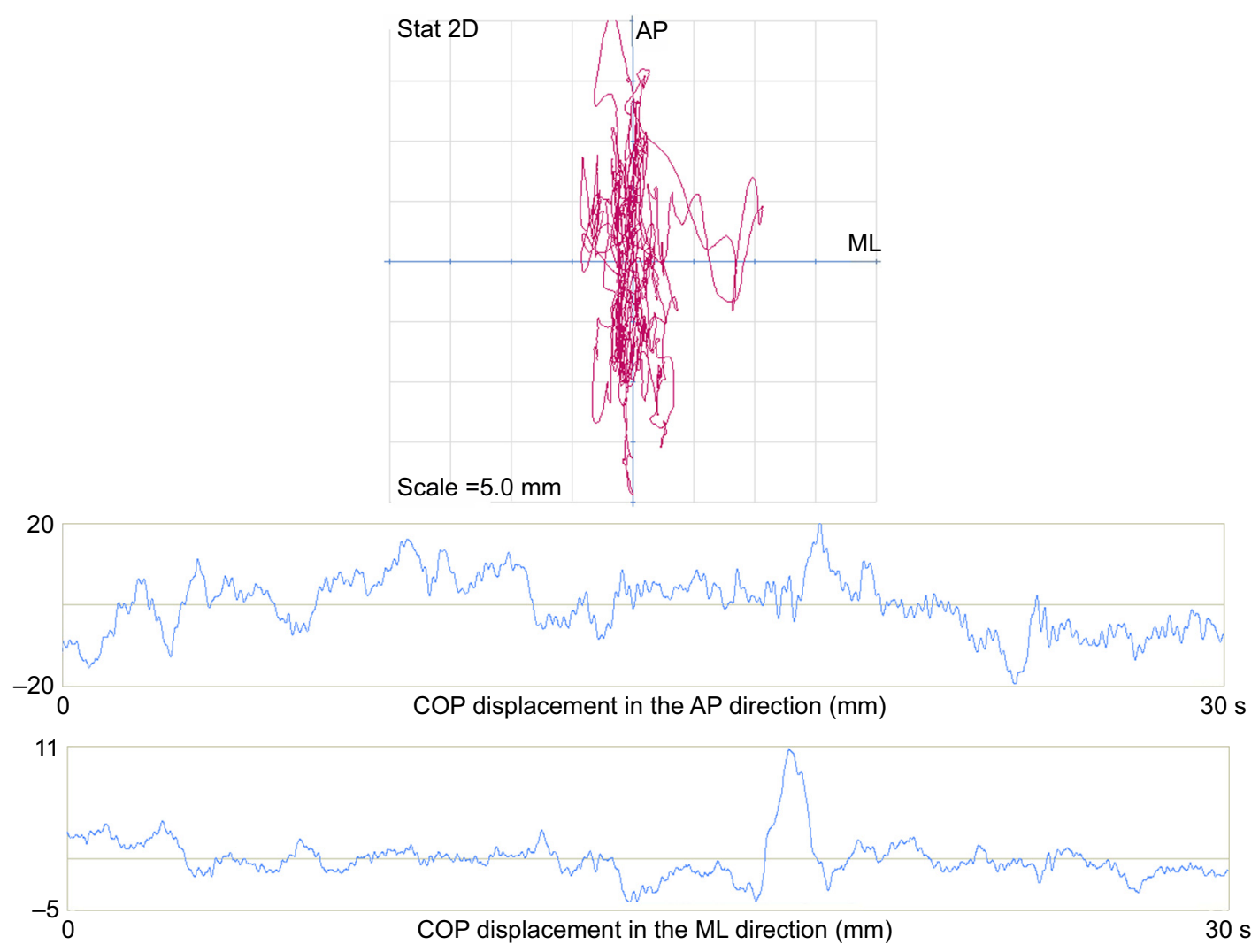

Figure 2 Diagram showing the sample of the path length for the COP during the test with the eyes closed. Abbreviations: AP, anteroposterior; COP, center of pressure; ML, mediolateral. 
Table I Somatic characteristics of the study subjects

\begin{tabular}{|c|c|c|c|c|c|}
\hline Variable & $\begin{array}{l}\text { Age, } \\
\text { years }\end{array}$ & Women & Men & $t$ & $p$-value ${ }^{a}$ \\
\hline \multirow[t]{3}{*}{ Body weight, kg } & $60-69$ & $77.54 \pm 17.14$ & $77.78 \pm 8.58$ & -0.05 & 0.962 \\
\hline & 70-79 & $70.53 \pm 14.22$ & $80.07 \pm \mid 3.11$ & -2.36 & $0.023^{b}$ \\
\hline & $80-90$ & $62.95 \pm 12.44$ & $80.00 \pm 12.29$ & -5.36 & $<0.00 \mathrm{I}^{\mathrm{b}}$ \\
\hline \multirow[t]{3}{*}{ Body height, cm } & $60-69$ & $158.76 \pm 8.95$ & $169.86 \pm 7.84$ & -3.63 & $0.00 \mathrm{I}^{\mathrm{b}}$ \\
\hline & $70-79$ & $157.93 \pm 8.67$ & $169.40 \pm 4.72$ & -4.84 & $<0.001^{b}$ \\
\hline & $80-90$ & $155.14 \pm 7.10$ & $166.82 \pm 4.78$ & -7.02 & $<0.00 \mathrm{I}^{\mathrm{b}}$ \\
\hline \multirow[t]{3}{*}{ BMI } & $60-69$ & $30.60 \pm 5.70$ & $27.15 \pm 3.19$ & 2.03 & 0.051 \\
\hline & 70-79 & $28.31 \pm 5.40$ & $27.73 \pm 3.69$ & 0.17 & 0.866 \\
\hline & $80-90$ & $26.50 \pm 6.47$ & $28.52 \pm 3.92$ & -1.35 & 0.180 \\
\hline
\end{tabular}

Notes: Data show mean $\pm S D$. ${ }^{a} p$-values were calculated using the Student's $t$-test for independent samples; ${ }^{b} p<0.05$.

Abbreviations: BMI, body mass index; $t$, value of the Student's $t$-test statistic.

somatic characteristics of the study subjects are presented in Table 1.

In order to ensure overall integrity of the research process, all tests were carried out in the morning, using the same measuring instruments operated by the authors. The measurements were carried out in a gym, in the conditions facilitating elimination of any acoustic stimuli that might have interfered with postural reflexes during the study. The seniors were barefooted, dressed in sportswear.

Consistency of the values characterized by a normal distribution was verified using the Shapiro-Wilk test. Parametric Student's $t$-test for independent samples was applied to analyze the data with normal distribution, while the non-parametric Mann-Whitney $U$-test was used due to the non-compliance with the timetable of a normal distribution. The results were considered statistically significant, if the probability level of the test was lower than the predetermined significance level $p<0.05$. The Stat Soft STATISTICA application (version 10.0) was used to process the test results.

\section{Ethics approval and informed consent}

The study protocol was approved by the Bioethics Review Committee, University of Rzeszow (Approval Ref No 11/02/2013). All procedures were carried out in full compliance with the Declaration of Helsinki. All study participants gave their written informed consent to participate in the study protocol.

\section{Results}

The data presented in Table 2 indicate that, in the open-eye test, the mean values of COP displacement in men were higher. However, statistically significant sex-related differences were observed in the case of the ML statokinesiogram path (SPML) - in the 60-69 years age group ( $p=0.004)$, the mean frequency of COP displacements $(p=0.028)$ and their number in the ML direction ( $p=0.019)$ - in the $70-79$ years age group, and the mean number of COP displacements in the AP direction - in 80-90 years olds $(p=0.026)$.

The data in Table 3 reveal that - when the subjects were deprived of visual control - the mean values of indicators pertaining to COP displacements were higher for men than for women. Statistically significant gender-related differences in the 60-69 years age group were observed in terms of the mean frequency of COP displacements $(p=0.045)$ and the number of COP displacements in the AP direction ( $p=0.049$ ). In men aged 70-79 years, the statokinesiogram path in the AP direction was significantly longer than for women in the same age range $(p=0.015)$. There were no statistically significant gender-related differences in the length of the statokinesiogram path in the ML direction. Most of the statistically significant gender-related differences were observed in the oldest age group. Men were characterized by higher values of the statokinesiogram path length in the AP direction $(p=0.001)$, COP surface area $(p=0.003)$, mean COP displacement in the AP direction $(p=0.001)$, range of AP stability $(p<0.001)$, and range of ML stability $(p=0.048)$.

\section{Discussion}

Numerous studies indicate an age-related decrease in the ability to maintain a stable standing position. It is commonly acknowledged that deteriorating stability manifests itself, inter alia, in longer paths and the speed of COP displacements. This is indubitably owed to progressive involutionary changes in the anatomical and functional structures of the balance system, as well as to overall decrease in coordination capacity.

Blaszczyk et $\mathrm{al}^{28}$ indicates the 2 main causes of the worsening postural stability. First, the elderly are unable to estimate the optimum COP position as precisely as the young; second, the decrease in stability is due to increased COP oscillations resulting from the latency in receiving information about articular angles (mainly in the ankle joint). The author also takes due note of certain shifts in the seniors' postural strategies. The deficits of the vestibular system limit the use of the hip strategy, while the somatosensory limitations increase its significance.

At this point, it is worth considering whether involutionary changes in balance control affect women and men in equal terms. In order to improve overall safety and quality of life in this population group, it is essential to appreciate the key properties of the balance system.

Opinions ventured by different authors on the effect of sexual dimorphism on the balance control process are discrepant. Riva et $\mathrm{al}^{29}$ found the sex-related differences in 
Table 2 Values and relevance of the differences in the stability indexes, as recorded in the open-eye test in women and men, in all three age groups

\begin{tabular}{|c|c|c|c|c|c|c|c|c|}
\hline \multirow{2}{*}{$\begin{array}{l}\text { Age, } \\
\text { years }\end{array}$} & \multicolumn{3}{|l|}{ Women } & \multicolumn{3}{|l|}{ Men } & \multirow[t]{2}{*}{$Z$} & \multirow[t]{2}{*}{$p$-value ${ }^{a}$} \\
\hline & mean \pm SD & Me & QR & mean \pm SD & Me & QR & & \\
\hline \multicolumn{9}{|c|}{ SPAP-EO, mm } \\
\hline $60-69$ & $200.94 \pm 85.73$ & 164.00 & 123.00 & $297.07 \pm 189.32$ & 240.50 & 209.00 & $-1.4 \mid$ & 0.159 \\
\hline $70-79$ & $238.65 \pm 106.13$ & 198.00 & 135.00 & $282.53 \pm 121.37$ & 254.00 & 154.00 & -1.29 & 0.198 \\
\hline $80-90$ & $247.09 \pm 69.94$ & 242.00 & 113.00 & $300.50 \pm 103.20$ & 292.00 & 171.50 & -1.79 & 0.073 \\
\hline \multicolumn{9}{|c|}{ SPML-EO, mm } \\
\hline $60-69$ & $|27.4| \pm 33.83$ & 115.00 & 38.00 & $178.57 \pm 66.88$ & 158.50 & 71.00 & -2.84 & $0.004^{b}$ \\
\hline $70-79$ & $163.03 \pm 6 \mid .89$ & 141.00 & 55.00 & $160.87 \pm 60.29$ & 140.00 & 59.00 & 0.38 & 0.701 \\
\hline $80-90$ & $\mid 80.77 \pm 48.72$ & 172.00 & 76.00 & $170.35 \pm 50.90$ & 166.00 & 45.50 & 1.17 & 0.243 \\
\hline \multicolumn{9}{|c|}{ SA-EO, mm² } \\
\hline $60-69$ & $215.59 \pm 145.29$ & 180.00 & 121.00 & $506.28 \pm 455.83$ & 415.50 & 491.00 & -1.92 & 0.054 \\
\hline 70-79 & $377.72 \pm 330.70$ & 339.00 & 262.00 & $344.40 \pm 364.13$ & 196.00 & 279.00 & 0.54 & 0.586 \\
\hline $80-90$ & $352.05 \pm 230.80$ & 320.00 & 224.00 & $458.50 \pm 399.17$ & 365.00 & 154.50 & -1.48 & 0.138 \\
\hline \multicolumn{9}{|c|}{ MAAP-EO, mm } \\
\hline $60-69$ & $2.21 \pm 1.29$ & 2.00 & 0.80 & $3.13 \pm 1.83$ & 2.45 & 2.70 & -1.37 & 0.171 \\
\hline 70-79 & $2.88 \pm 1.25$ & 2.80 & 1.80 & $2.37 \pm 0.96$ & 2.50 & 1.30 & 1.26 & 0.207 \\
\hline $80-90$ & $2.42 \pm 0.99$ & 2.40 & 1.40 & $3.07 \pm 1.12$ & 3.10 & 1.40 & -2.23 & $0.026^{b}$ \\
\hline \multicolumn{9}{|c|}{ MAML-EO, mm } \\
\hline $60-69$ & $\mathrm{I} .2 \mathrm{I} \pm 0.73$ & 0.90 & 0.80 & $1.73 \pm 1.23$ & 1.20 & 1.20 & -1.23 & 0.218 \\
\hline $70-79$ & $1.67 \pm 1.33$ & 1.50 & 1.10 & $1.39 \pm 1.33$ & 0.90 & 1.20 & 1.30 & 0.194 \\
\hline $80-90$ & $1.6 \mathrm{I} \pm 1.07$ & 1.40 & 1.50 & $1.78 \pm 1.70$ & 1.50 & 1.10 & -0.49 & 0.626 \\
\hline \multicolumn{9}{|c|}{ MaxAP-EO, mm } \\
\hline $60-69$ & $8.40 \pm 4.16$ & 7.80 & 3.90 & II. $14 \pm 5.74$ & 9.60 & 8.90 & -1.05 & 0.293 \\
\hline $70-79$ & $10.60 \pm 5.29$ & 9.00 & 7.80 & $10.14 \pm 5.96$ & 8.00 & 6.00 & 0.47 & 0.638 \\
\hline $80-90$ & $9.99 \pm 5.43$ & 8.60 & 5.80 & $12.03 \pm 5.45$ & 11.00 & 7.50 & -1.68 & 0.094 \\
\hline \multicolumn{9}{|c|}{ MaxML-EO, mm } \\
\hline $60-69$ & $4.13 \pm 2.10$ & 3.30 & 3.10 & $6.47 \pm 3.85$ & 5.30 & 6.30 & -1.90 & 0.057 \\
\hline 70-79 & $6.73 \pm 8.03$ & 4.80 & 4.10 & $5.05 \pm 4.21$ & 3.60 & 5.70 & 1.27 & 0.202 \\
\hline $80-90$ & $6.22 \pm 4.14$ & 4.90 & 6.10 & $6.45 \pm 5.75$ & 5.15 & 3.75 & 0.12 & 0.906 \\
\hline \multicolumn{9}{|c|}{ MF-EO, $\mathrm{Hz}$} \\
\hline $60-69$ & $0.60 \pm 0.37$ & 0.51 & 0.31 & $0.60 \pm 0.26$ & 0.59 & 0.45 & -0.38 & 0.706 \\
\hline 70-79 & $0.53 \pm 0.25$ & 0.46 & 0.28 & $0.72 \pm 0.33$ & 0.59 & 0.47 & -2.19 & $0.028^{b}$ \\
\hline $80-90$ & $0.64 \pm 0.26$ & 0.57 & 0.33 & $0.58 \pm 0.27$ & 0.52 & 0.22 & 1.01 & 0.308 \\
\hline \multicolumn{9}{|c|}{ LWAP-EO } \\
\hline $60-69$ & $20.82 \pm \mid 6.91$ & 19.00 & 10.00 & $24.36 \pm 17.47$ & 22.50 & 13.00 & -0.93 & 0.351 \\
\hline $70-79$ & $20.03 \pm 13.77$ & 19.00 & 16.00 & $30.60 \pm 19.30$ & 25.00 & 18.00 & -1.93 & 0.053 \\
\hline $80-90$ & $23.21 \pm 12.43$ & 20.00 & 18.00 & $26.95 \pm 13.36$ & 23.00 & 17.50 & -1.26 & 0.209 \\
\hline \multicolumn{9}{|c|}{ LWML-EO } \\
\hline $60-69$ & $|5.12 \pm 9.8|$ & 15.00 & 10.00 & $|8.2| \pm 10.46$ & 20.00 & 13.00 & -0.97 & 0.331 \\
\hline $70-79$ & $|3.79 \pm 7.7|$ & 14.00 & 14.00 & $22.53 \pm 12.83$ & 23.00 & 16.00 & -2.34 & $0.019^{b}$ \\
\hline $80-90$ & $|8.63 \pm| 2.2 \mid$ & 16.00 & 13.00 & $18.15 \pm 10.00$ & 19.00 & 9.50 & -0.34 & 0.734 \\
\hline
\end{tabular}

Notes: a $p$-values were calculated using the Mann-Whitney U-test; ${ }^{b} p<0.05$.

Abbreviations: COP, center of pressure; EO, eyes open; LWAP, number of COP displacements in the anteroposterior direction; LWML, number of COP displacements in the mediolateral direction; MAAP, mean COP displacement in the anteroposterior direction; MAML, mean COP displacement in the mediolateral direction; MaxAP, range of anteroposterior stability; MaxML, range of mediolateral stability; Me, median; MF, mean frequency of COP displacement; QR, quartile range; SA, sway area delimited by the COP point; SPAP, statokinesiogram path length in the anteroposterior direction; SPML, statokinesiogram path length in the mediolateral direction; $Z$, value of the Mann-Whitney U-test statistic.

stability index (SI) values measured both with and without visual control. Women had significantly poorer balance than men. However, there were no such differences in a younger age category that they also investigated (65-74 years). The authors attribute the greater deterioration of single-stance stability in older women predominantly to more accentuated proprioceptive decay. Kim et a ${ }^{30}$ observed a clear deterioration in SIs (increased COP displacement velocity and overall reaction force) in seniors, especially along the ML direction, with marked gender-related differences. On the other hand, in their studies of men and women aged 20-70 years, Røgind et $\mathrm{al}^{31}$ found no differences in postural stability between 
Table 3 Values and relevance of the differences in the stability indexes, as recorded in the closed-eye test, in the separate groups of women and men

\begin{tabular}{|c|c|c|c|c|c|c|c|c|}
\hline \multirow{2}{*}{$\begin{array}{l}\text { Age, } \\
\text { years }\end{array}$} & \multicolumn{3}{|l|}{ Women } & \multicolumn{3}{|l|}{ Men } & \multirow[t]{2}{*}{$\mathbf{Z}$} & \multirow[t]{2}{*}{ p-value ${ }^{a}$} \\
\hline & mean \pm SD & Me & QR & mean \pm SD & Me & QR & & \\
\hline \multicolumn{9}{|c|}{ SPAP-EC, mm } \\
\hline $60-69$ & $286.35 \pm I 18.85$ & 258.00 & 227.00 & $435.7 I \pm 287 . I I$ & 302.50 & 410.00 & -1.21 & 0.226 \\
\hline $70-79$ & $379.4 I \pm 194.63$ & 289.00 & 189.00 & $527.60 \pm 265.46$ & 416.00 & 322.00 & -2.43 & $0.015^{b}$ \\
\hline $80-90$ & $311.18 \pm 115.84$ & 287.00 & 160.00 & $481.20 \pm 202.63$ & 434.50 & 327.00 & -3.44 & $0.00 \mathrm{I}^{\mathrm{b}}$ \\
\hline \multicolumn{9}{|c|}{ SPML-EC, mm } \\
\hline $60-69$ & I $58.88 \pm 54.59$ & 153.00 & 82.00 & $217.28 \pm 92.23$ & 192.00 & 176.00 & -1.63 & 0.104 \\
\hline $70-79$ & $188.45 \pm 68.34$ & 173.00 & 75.00 & $204.87 \pm 104.10$ & 157.00 & 74.00 & -0.17 & 0.862 \\
\hline $80-90$ & $195.50 \pm 57.88$ & 183.00 & 71.00 & $230.30 \pm 103.56$ & 212.00 & 100.50 & -0.96 & 0.337 \\
\hline \multicolumn{9}{|c|}{ SA-EC, $\mathrm{mm}^{2}$} \\
\hline $60-69$ & $402.47 \pm 235.72$ & 389.00 & 346.00 & $632.50 \pm 663.33$ & 361.50 & 639.00 & -0.55 & 0.578 \\
\hline $70-79$ & $575.17 \pm 482.42$ & 382.00 & 431.00 & $643.47 \pm 400.33$ & 556.00 & 575.00 & -1.01 & 0.310 \\
\hline $80-90$ & $441.50 \pm 320.90$ & 321.00 & 297.00 & $823.50 \pm 625.60$ & 615.50 & 581.00 & -3.02 & $0.003^{b}$ \\
\hline \multicolumn{9}{|c|}{ MAAP-EC, mm } \\
\hline $60-69$ & $3.39 \pm 1.78$ & 2.90 & 2.40 & $3.49 \pm 2.32$ & 3.10 & 2.00 & 0.10 & 0.921 \\
\hline $70-79$ & $3.61 \pm 1.62$ & 3.30 & 1.90 & $4.12 \pm 1.78$ & 3.70 & 1.90 & -1.05 & 0.293 \\
\hline $80-90$ & $2.86 \pm 1.39$ & 2.60 & 1.20 & $4.48 \pm 1.95$ & 3.70 & 3.55 & -3.31 & $0.00 \mathrm{I}^{\mathrm{b}}$ \\
\hline \multicolumn{9}{|c|}{ MAML-EC, mm } \\
\hline $60-69$ & $1.64 \pm|.2|$ & 1.30 & 0.80 & $1.48 \pm 1.03$ & 1.25 & 0.70 & 0.40 & 0.691 \\
\hline $70-79$ & $1.57 \pm 1.03$ & 1.50 & 1.20 & $1.49 \pm 0.65$ & 1.30 & 0.70 & -0.21 & 0.833 \\
\hline $80-90$ & $1.59 \pm 1.11$ & 1.20 & 1.00 & $1.97 \pm 1.19$ & 1.85 & 1.10 & -1.89 & 0.058 \\
\hline \multicolumn{9}{|c|}{ MaxAP-EC, mm } \\
\hline $60-69$ & $12.38 \pm 5.17$ & 10.80 & 7.10 & $13.85 \pm 7.09$ & 13.05 & 7.20 & -0.50 & 0.620 \\
\hline $70-79$ & $5.39 \pm 7.50$ & 12.80 & 8.50 & $16.57 \pm 7.90$ & 16.10 & 14.40 & -0.25 & 0.804 \\
\hline $80-90$ & II. $44 \pm 5.08$ & 9.50 & 6.90 & $19.57 \pm 10.39$ & 15.40 & 14.40 & -3.72 & $<0.00 \mathrm{I}^{\mathrm{b}}$ \\
\hline \multicolumn{9}{|c|}{ MaxML-EC, mm } \\
\hline $60-69$ & $7.90 \pm 6.44$ & 5.40 & 5.70 & $8.56 \pm 10.78$ & 5.25 & 6.20 & 0.32 & 0.751 \\
\hline $70-79$ & $6.44 \pm 4.63$ & 1.50 & 1.20 & $5.77 \pm 3.52$ & 4.70 & 3.70 & 0.28 & 0.776 \\
\hline $80-90$ & $6.10 \pm 4.50$ & 4.80 & 4.00 & $8.03 \pm 5.26$ & 6.50 & 4.90 & -1.99 & $0.048^{\mathrm{b}}$ \\
\hline \multicolumn{9}{|c|}{ MF-EC, $\mathrm{Hz}$} \\
\hline $60-69$ & $0.56 \pm 0.29$ & $0.5 I$ & 0.21 & $0.73 \pm 0.32$ & 0.65 & 0.31 & -2.00 & $0.045^{b}$ \\
\hline $70-79$ & $0.64 \pm 0.32$ & 0.51 & 0.35 & $0.72 \pm 0.27$ & 0.69 & $0.4 I$ & -1.46 & 0.144 \\
\hline $80-90$ & $0.66 \pm 0.22$ & 0.64 & 0.30 & $0.64 \pm 0.26$ & 0.60 & 0.33 & 0.50 & 0.605 \\
\hline \multicolumn{9}{|c|}{ LWAP-EC } \\
\hline $60-69$ & $23.70 \pm 17.08$ & 20.00 & 16.00 & $32.78 \pm 20.11$ & 23.00 & 26.00 & -1.49 & 0.137 \\
\hline $70-79$ & $28.62 \pm 18.14$ & 23.00 & 20.00 & $33.80 \pm 21.31$ & 28.00 & 26.00 & -0.94 & 0.347 \\
\hline $80-90$ & $26.23 \pm 13.17$ & 25.00 & 13.00 & $30.90 \pm 15.73$ & 32.50 & 17.50 & -1.40 & 0.160 \\
\hline \multicolumn{9}{|c|}{ LWML-EC } \\
\hline $60-69$ & $|8.94 \pm| 3.5 \mid$ & 14.00 & 20.00 & $29.36 \pm|5.5|$ & 27.00 & 18.00 & -1.96 & $0.049^{b}$ \\
\hline $70-79$ & $22.14 \pm 10.58$ & 22.00 & 15.00 & $24.27 \pm 12.66$ & 23.00 & 17.00 & -0.32 & 0.747 \\
\hline $80-90$ & $21.42 \pm 12.30$ & 21.00 & 15.00 & $26.15 \pm 16.38$ & 23.00 & 23.50 & -0.86 & 0.392 \\
\hline
\end{tabular}

Notes: a $p$-values were calculated using the Mann-Whitney $U$-test; ${ }^{b} p<0.05$.

Abbreviations: COP, center of pressure; MAAP, mean COP displacement in the anteroposterior direction; EC, eyes closed; LWAP, number of COP displacements in the anteroposterior direction; LWML, number of COP displacements in the mediolateral direction; MAML, mean COP displacement in the mediolateral direction; MaxAP, range of anteroposterior stability; MaxML, range of mediolateral stability; Me, median; MF, mean frequency of COP displacement; QR, quartile range; SA, sway area delimited by the COP point; SPAP, statokinesiogram path length in the anteroposterior direction; SPML, statokinesiogram path length in the mediolateral direction; Z, value of the Mann-Whitney U-test statistic.

the sexes. The authors observed an age-related reduction of the use of ankle strategy, as the subjects' body weight increased. Hue et al ${ }^{32}$ reported a statistically significant negative correlation between body weight and stability indicators, which they attributed to the reduced sensitivity of kinesthetic and tactile receptors in overweight individuals.
Gender-related differences in the values of stability indicators were also observed by Skalska et $\mathrm{al}^{24}$ and Stemplewski et al. ${ }^{33}$ The authors found a significantly longer stabilogram path and an increase in the rate of corrective responses in the AP direction in men under conditions involving the use of foam pads. Blaszczyk et $\mathrm{al}^{28}$ observed the correlation between the 
speed of COP displacements along the AP direction with the frequency of falls among the elderly. The authors also noted the increase in lower limb performance asymmetry related to an increase in stabilogram path length, which they believed was an adaptation to less stable conditions.

In the present study, the mean values of stabilogram indicators observed for men were higher than those measured for women in the corresponding age groups. This is due to the fact that men are characterized by a lower level of postural stability when compared with women. Changing the test conditions by way of limiting input visual information resulted in an increase in the stabilogram values observed for both sexes, with the statistically significant differences noted in the most advanced age groups. In men, a longer path of the statokinesiogram in the AP direction and higher values of average COP displacements in the AP direction were found. These differences may stem from the distinct postural strategies adopted by men and women in response to the deterioration of the mechanisms of body regulation and control associated with aging.

An increased rate of the so-called "swaying", as observed in men during a quiet stance, may well be correlated with respective values of the somatic features. Men were characterized by significantly higher values of body height, and thus higher positioning of COG in relation to the plane of support. Comparison of the mechanism of balance control to an inverted pendulum, whereby the main axis of rotation is provided by the ankle joint, gives grounds to conclude that the higher the COG is positioned, the harder it is to have it effectively stabilized, hence greater problems in balance control are encountered in men. The study of Bryant et al, ${ }^{34}$ which reveals a lack of variation in the extent of swaying in both sexes, following the necessary data adjustment for height, gives extra credence to this particular course of reasoning.

Blaszczyk et $\mathrm{al}^{28}$ highlighted that increased postural tilts in the standing position may be aggravated in view of the progressive neuromuscular impairment, which results in the increased activity thresholds of the sensory systems in the elderly, as well as in the increased, uncontrolled muscle stimulation. Juxtaposing these observations against our own results, it may well be assumed that men are more susceptible to the degradation of somatosensory systems within the foot and ankle joint, as well as manifest a greater tendency toward joint stiffness and muscle involution.

The potential causes of differences in balance control may also be looked upon in terms of the surface of the support plane. Assuming that men have larger feet, and hence a larger support plane, it follows that they should be able to cope much better with greater COP variability. Our previous study showed, however, that only in the case of women, longer and wider feet provided better stability. In turn, in men, the length and width of the feet were not the predictive variables for the stability indicators, whereas the deformations of the feet, for example, the lowered longitudinal arch and hallux valgus, were found to actually affect stability. ${ }^{35}$

The results yielded by the present study reveal that the gender-related differences in automated postural responses do occur. It might then be concluded that the consequences of aging in relation to postural stability may differ between men and women. Under the conditions involving destabilization, especially in the face of progressive muscle failure, men are found to be more prone to a loss of balance. The results yielded by our study indicate the need to have the gender-related differences taken into account, when developing various preventive and therapeutic programs, as well as mapping out further research into the subject. It is also an essential conceptual premise to be borne in mind when giving specific advice on enhancing postural balance, as well as on structuring postural and proprioceptive reeducation programs in seniors. Especially in the case of men, physical rehabilitation programs should comprise the exercises aimed at preventing degradation of somatosensory systems within the foot and ankle joint, as well as joint stiffness and muscle involution. Training should, therefore, make use of both static exercises and dynamic tasks that reduce the support plane by changing the foot position. Exercises on unstable platforms and movement tasks with a visual control of body positioning withheld are also of major significance. The recommendations, as presently outlined, seem to hold appreciable potential in terms of improving postural balance and overall functional capacity in the elderly.

The results yielded by our own research do not provide sufficient grounds, however, to make any inferences on the actual causes of gender-related differences in postural stability. Nevertheless, they do offer a certain promising input that might inspire further research into the trends observed to date. In the authors' view, any such continuing research should best be focused on identifying the specific factors and mechanisms that interfere with postural balance control in elderly women and men.

\section{Study limitations}

The way the subjects were admitted into the study, pursued in line with the adopted inclusion criteria, on the one hand, allowed homogeneity within a group to be ensured, fully corresponding to pertinent characteristics of a seniors' 
population, while on the other, resulted in reducing the number of potential recruits, which might well be regarded as a study limitation.

\section{Conclusion}

Gender-related differences affecting postural stability were found in the elderly. Men were characterized by a lower level of postural stability when compared with women. This highlights the need to have the gender-related differences taken into account, when developing various preventive and therapeutic programs specifically aimed at compensating certain involution-dependent deficits. Arguably, specific results yielded by the present study are encouraging enough to warrant further research endeavors into the subject matter.

\section{Author contributions}

EPL: conceptualization, data curation, formal analysis, investigation, methodology, project administration, resources, supervision, validation, writing - original draft, writing review and editing. PB: data curation, formal analysis, project administration, resources, software, validation, visualization. SJ: funding acquisition, methodology, software, supervision, visualization. JO: data curation, project administration, resources, software. MZ: conceptualization, formal analysis, funding acquisition, investigation, methodology, supervision, validation, visualization, writing - original draft, writing - review and editing. All authors contributed toward data analysis, drafting and revising the paper and agree to be accountable for all aspects of the work.

\section{Disclosure}

The authors report no conflicts of interest in this work.

\section{References}

1. Chen MA, Kawakubo M, Colletti PM, et al. Effect of age on aortic atherosclerosis. J Geriatr Cardiol. 2013;10(2):135-140.

2. Biskup M, Gozdz S, Macek P, et al. Falls-risk in senior women after radical treatment of breast cancer. Med Stud. 2017;33(2):110-116.

3. Doherty TJ. Invited review: aging and sarcopenia. J Appl Physiol (1985). 2003;95(4):1717-1727.

4. Al-Momani M, Al-Momani F, Alghadir AH, Alharethy S, Gabr SA. Factors related to gait and balance deficits in older adults. Clin Interv Aging. 2016;11:1043-1049.

5. Vaughan L, Corbin AL, Goveas JS. Depression and frailty in later life: a systematic review. Clin Interv Aging. 2015;10:1947-1958.

6. Zak M, Krupnik S, Puzio G, Staszczak-Gawelda I, Czesak J. Assessment of functional capability and on-going falls-risk in older institutionalized people after total hip arthroplasty for femoral neck fractures. Arch Gerontol Geriatr. 2015;61(1):14-20.

7. Hsu WL, Chen CY, Tsauo JY, Yang RS. Balance control in elderly people with osteoporosis. J Formos Med Assoc. 2014;113(6):334-339.

8. Cameron ID, Gillespie LD, Robertson MC, et al. Interventions for preventing falls in older people in care facilities and hospitals. Cochrane Database Syst Rev. 2012;12:CD005465.
9. Melton LJ. Who has osteoporosis? A conflict between clinical and public health perspectives. J Bone Miner Res. 2000;15(2):2309-2314.

10. Francis RM. Fallus and fractures. Age Ageing. 2001;30(4):25-28.

11. Naessen T, Lindmark B, Larsen HC. Hormone therapy and postural balance in elderly women. Menopause. 2007;14(6):1020-1024.

12. Otero M, Esain I, González-Suarez ÁM, Gil SM. The effectiveness of a basic exercise intervention to improve strength and balance in women with osteoporosis. Clin Interv Aging. 2017;12:505-513.

13. Liu-Ambrose T, Eng JJ, Khan KM, Mallinson A, Carter ND, McKay HA. The influence of back pain on balance and functional mobility in 65 - to 75 -year-old women with osteoporosis. Osteoporos Int. 2002;13(11): 868-873.

14. Ishikawa Y, Miyakoshi N, Kasukawa Y, Hongo M, Shimada Y. Spinal curvature and postural balance in patients with osteoporosis. Osteoporos Int. 2009;20(12):2049-2053.

15. Campbell AJ, Borrie MJ, Spears GF. Risk factors for falls in a community-based prospective study of people 70 years and older. J Gerontol. 1989;44(4):M112-M117.

16. Frontera WR, Hughes VA, Lutz KJ, Evans WJ. A cross-sectional study of muscle strength and mass in 45- to 78-year-old men and women. J Appl Physiol (1985). 1991;71(2):644-650.

17. Cooper R, Hardy R, Aihie Sayer A, et al; HALCyon study team. Age and gender differences in physical capability levels from mid-life onwards: the harmonisation and meta-analysis of data from eight UK cohort studies. PLoS One. 2011;6(11):e27899.

18. Menant JC, Steele JR, Menz HB, Munro BJ, Lord SR. Effects of footwear features on balance and stepping in older people. Gerontology. 2008; $54(1): 18-23$.

19. Cronin NJ, Barrett RS, Carty CP. Long-term use of high-heeled shoes alters the neuromechanics of human walking. J Appl Physiol (1985). 2012;112(6):1054-1058.

20. Kado DM, Huang MH, Karlamangla AS, Barrett-Connor E, Greendale GA. Hyperkyphotic posture predicts mortality in older community-dwelling men and women: a prospective study. J Am Geriatr Soc. 2004;52(10): $1662-1667$

21. Takahashi T, Ishida K, Hirose D, et al. Trunk deformity is associated with a reduction in outdoor activities of daily living and life satisfaction in community-dwelling older people. Osteoporos Int. 2005;16(3): 273-279.

22. Sinaki M, Brey RH, Hughes CA, Larson DR, Kaufman KR. Balance disorder and increased risk of falls in osteoporosis and kyphosis: significance of kyphotic posture and muscle strength. Osteoporos Int. 2005; 16(8):1004-1010

23. Perrin P, Deviterne D, Hugel F, Perrot C. Judo, better than dance, develops sensorimotor adaptabilities involved in balance control. Gait Posture. 2002;15(2):187-194.

24. Skalska A, Ocetkiewicz T, Zak M, Grodzicki T. Influence of age on the parameters of postural control measured with balance platform. New Med. 2004;7(1):12-16.

25. Swanenburg J, de Bruin ED, Favero K, Uebelhart D, Mulder T. The reliability of postural balance measures in single and dual tasking in elderly fallers and non-fallers. BMC Musculoskelet Disord. 2008; 9:162.

26. Kim GT, Ferdjallah M, Harris GF. Fast computational analysis of sway area using center of pressure data in normal children and children with cerebral palsy. Am J Biomed Sci. 2009;1(4):364-372.

27. Hill MW, Higgins MF, Price MJ. The effect of high-intensity cycling training on postural sway during standing under rested and fatigued conditions in healthy young adults. Eur J Appl Physiol. 2016;116(10): 1965-1974.

28. Blaszczyk JW, Prince F, Raiche M, Hébert R. Effect of ageing and vision on limb load asymmetry during quiet stance. $J$ Biomech. 2000;33(10): $1243-1248$.

29. Riva D, Mamo C, Fanì M, et al. Single stance stability and proprioceptive control in older adults living at home: gender and age differences. J Aging Res. 2013;2013:561695. 
30. Kim JW, Eom GM, Kim CS, et al. Sex differences in the postural sway characteristics of young and elderly subjects during quiet natural standing. Geriatr Gerontol Int. 2010;10(2):191-198.

31. Røgind H, Lykkegaard JJ, Bliddal H, Danneskiold-Samsøe B. Postural sway in normal subjects aged 20-70 years. Clin Physiol Funct Imaging. 2003;23(3):171-176.

32. Hue O, Simoneau M, Marcotte J, et al. Body weight is a strong predictor of postural stability. Gait Posture. 2007;26(1):32-38.

33. Stemplewski R, Salamon A, Maciaszek J. Static body balance posturographic values in medio-lateral and anterior-posterior directions in conditions of unstable base of support in men older than 70 years. Stud Phys Cult Tourism. 2006;13:85-87.
34. Bryant EC, Trew ME, Bruce AM, Kuisma RM, Smith AW. Gender differences in balance performance at the time of retirement. Clin Biomech (Bristol, Avon). 2005;20(3):330-335.

35. Puszczalowska-Lizis E, Bujas P, Omorczyk J, Jandzis S, Zak M. Feet deformities are correlated with impaired balance and postural stability in seniors over 75. PLoS One. 2017;12(9):e0183227.

\section{Publish your work in this journal}

Clinical Interventions in Aging is an international, peer-reviewed journal focusing on evidence-based reports on the value or lack thereof of treatments intended to prevent or delay the onset of maladaptive correlates of aging in human beings. This journal is indexed on PubMed Central, MedLine,

\section{Dovepress}

CAS, Scopus and the Elsevier Bibliographic databases. The manuscript management system is completely online and includes a very quick and fair peer-review system, which is all easy to use. Visit http://www.dovepress. com/testimonials.php to read real quotes from published authors. 